\title{
Opium Addiction and Mortality Among Burn Patients
}

\author{
Mohammad Motamedolshariati, ${ }^{1}$ Amir Mirhaghi, ${ }^{2}$ and Seyed Hassan Tavousi ${ }^{3,}$ \\ ${ }^{1}$ Vascular and Endovascular Surgery Research Center, Imam Reza Hospital, Faculty of Medicine, Mashhad University of Medical Sciences, Mashhad, IR Iran \\ ${ }^{2}$ Evidence-Based Caring Research Center, School of Nursing and Midwifery, Mashhad University of Medical Sciences, Mashhad, IR Iran \\ ${ }^{3}$ Surgical Oncology Research Center, Imam Reza Hospital, Faculty of Medicine, Mashhad University of Medical Sciences, Mashhad, IR Iran \\ "Corresponding author: Seyed Hassan Tavousi, Surgical Oncology Research Center, Imam Reza Hospital, Faculty of Medicine, Mashhad University of Medical Sciences, \\ Mashhad, IR Iran. Tel: +98-5138022677, Fax: +98-5138525255, E-mail: tavoosih@mums.ac.ir
}

Received 2015 March 09; Revised 2015 September 15; Accepted 2015 September 19.

\begin{abstract}
Background: Opium addiction is a major issue amongst burn-patients in Iran. A few studies have addressed interactions between burn and addiction.

Objectives: The aim of the present study was to compare mortality and morbidity between addicted and non-addicted patients with burn injuries.

Patients and Methods: This research was a prospective paired matched-cohort study that was conducted from October 2012 to July 2013 at the Burn Unit of Mashhad University of Medical Sciences. One hundred and fifty-two burn patients (64\% of 237) were matched in pairs based on age, gender, total body surface area and burn thickness. Mortality and morbidity was compared between the two groups using the McNemar test, the conditional logistic regression model and the Kaplan-Meier method.

Results: Fourteen (9.2\%) patients died at the Burn unit. McNemar test found statistically significant differences in mortality between the two groups. Survival analysis using the Kaplan-Meier method revealed that the estimated mean survival time was 59.02 (CI $95 \%$ : 41.83 - 76.21) for non-addicted patients and 118.78 (CI 95\%: 106.52 - 131.04) for addicted patients. The odds ratio for mortality was 0.524 (CI 95\%: 0.167 -1.64) times greater for addicted patients compared with non-addicted patients.

Conclusions: Addiction in burn patients could be considered as a factor against mortality that deserves further studies. Besides, addicted patients didn't show significantly higher morbidity than non-addicted patients.
\end{abstract}

Keywords: Burns, Substance-Related Disorders, Survival, Mortality

\section{Background}

Burn injuries are life-threatening events especially in developing countries, and cause substantial morbidity and mortality (1-3). Burns are also one of the most expensive traumatic injuries, because of the long length of stay in hospitals and relevant complications (4).

Burn injuries could be accompanied by several risk factors, which increase morbidity and mortality but few studies have considered identifying the effects of specific comorbidities on outcome $(5,6)$. Some studies have investigated the influence of risk factors on survival of burn patients with alcohol abuse because alcohol consumption is more common in western countries, yet burn patients with other kinds of dependency such as opium addiction needs more investigations. Burn patients with alcohol abuse have shown more morbidity than others (7). Opium addiction has been considered as a risk factor in a human's life yet it is not clear how much it is of a risk factor for a burn patient.

Drug dependency among burn patients at the Burn Unit (BU) is a major issue, especially in $\operatorname{Iran}(8,9)$. It has been estimated that there are three million individuals with drug dependency in Iran (10). Opium smoking is the most common form of substance abuse used by substancedependent people (8). Statistics have revealed a drug dependency rate among burn patients varying from 10 to $30 \%$ in Iran, which is far from what has been reported in the U.S. $(3.3 \%)(6,8,11)$. On the contrary, one study on a burn intensive care unit reported that drug addiction was not commonly associated with burns. It is expected for this study to show relatively different epidemiological statistics compared to studies on burn patients at BU(11). Generally, studies have indicated that the rate of burn events could vary geographically (12). A few studies have investigated opium addiction among burn patients, who were admitted to the Burn Unit (BU) $(8,9)$. Studies cautiously stated that burn patients with drug abuse have shown more morbidity than others (13).

In addition, our long-term observations have indicated that addicted patients are substantially resistant to worse outcomes and they also have higher survival rate compared to non-addicted patients, which is marginally supported by a few studies (11). Controversial results suggest more in-depth exploration into the interaction between burn and addiction. 


\section{Objectives}

The aim of this study was to compare mortality and morbidity between addicted and non- addicted patients with burn injury.

\section{Patients and Methods}

This research was a prospective paired matched-cohort study. Admitted patients were divided into two groups on the basis of addiction, including those who are addicted (exposed) and non-addicted (unexposed). The study was conducted from October 2012 to July 2013 at a BU. Ethical approval for the study was obtained from the University's ethics committee that required patients to sign a consent form. All patients were given detailed explanations and they signed an informed consent.

\subsection{Subjects}

Patients were entered in the study based on the inclusion criteria and then they were matched individually on the basis of age, gender, burn causes, Total Body Surface Area (TBSA), burn thickness, time of burn injury and comorbid medical history. Female opioid-dependent patients were rare in the $\mathrm{BU}$, therefore only male patients were included. The unmatched study population was a convenience sample of all male patients who were admitted at the BU based on the inclusion criteria. They were observed and their data was recorded. Two equal and similar groups that were only different in terms of substance dependency were derived from the unmatched group. One non-addicted individual in the unexposed group was matched to each corresponding addicted patient. Patients were eligible for inclusion in the study if they had been admitted within 24 hours of initial burn injury due to thermal burn as well as patients who had preexisting co-morbidities or multiple injuries. The following cases were excluded from the study: patients who were admitted dead on arrival or, transferred inter-institutionally as well as patients who received extraordinary treatment. Incomplete medical records were also excluded.

\subsection{Settings}

The BU at Imam Reza hospital located of Mashhad (Iran) is responsible for providing specialized and expert burn care for all burn victims, who were referred from the east of Iran. Therefore, it is an excellent facility for conducting an epidemiologic study. Each year 900 and 4000 patients are admitted to the inpatient and outpatient unit, respectively.

\subsection{Data Collection}

Data were collected from patients, medical records during field visits. Addicted patients were interviewed by an expert psychologist, regarding substance dependency. She interviewed all patients to determine substancedependent individuals. Substance dependency was identified using the Diagnostic and statistical manual of mental disorders (DSM)-V criteria for substance use disorders.

Demographic variables such as age, gender, marital status, employment status and residency were examined as well as Length of Stay (LOS), admission and disposition date. Among substance-dependent individuals, pattern of substance dependency including duration, extent, severity and type of substance use, psychoactive medication history, suicide history and crime history were also recorded. Burn injury characteristics including time of injury, burn cause, Total Burn Surface Area (TBSA), burn thickness and inhalation burn injury as well as burn surgery and debridement were assessed for keeping homogeneity. Mortality and morbidity outcomes such as wound culture result, ventilator support, cardiopulmonary resuscitation (CPR) attempts, intensive care unit (ICU) admission, and blood transfusion were also examined.

\subsection{Statistical Analysis}

Comparison of the two matched groups was performed using descriptive statistics to report central tendency statistics (mean and standard deviation) as well as bivariate analysis to identify the variables that had significant associations between the two matched groups. McNemar test was used to compare categorical variables (mortality, marital status, employment, crime history, addiction history, psychiatric medication history, suicide history, full-thickness burn, inhalation burn, domestic gas, flame, cardiopulmonary resuscitation (CPR) attempts, substance dependency). Paired t-test was applied to compare continuous variables (age, TBSA, length of stay, intensive care unit (ICU) stays, transfusion units and ventilator support). The conditional logistic regression (CLR) model to control possible confounders affecting mortality was used. The CLR permits the use of both continuous variables and categorical variables. Survival analysis using the KaplanMeier curve of survival was applied for the addicted and non-addicted groups (14). Data analysis was performed using the SPSS software, version 15.0 (SPSS Inc., Chicago, Illinois). Aside from analysis of matched data, all admitted male burn patients during the observational period (October 2012-July 2013) were analyzed as the unmatched group in the BU. In fact, the current study reported two sets of measured data. The first dataset, included 152 patients organized in matched groups, and the second data set in- 
cluded 237 burn patients (unmatched group), that composed of 152 matched patients and another 85 patients that couldn't be paired into matched groups. Statistical power analysis was conducted by $\mathrm{G}^{*}$ power 3.1. A post-hoc power analysis was completed to determine the study power according to mortality proportions $(\pi 11=0.03, \pi 12=0.46$, $\pi 21=0.06$ and $\pi 22=0.44$ ) using inequality, two dependent groups (McNemar) method, so a power coefficient of 0.99 was achieved.

\section{Results}

Two hundred and fifty-one male patients were admitted during the observational period. Fourteen patients were excluded because of graft surgery (4), electrical burn (7), toxic epidermal necrolysis (2) and incomplete documentation (1), thus 237 male patients were left as the unmatched group. One hundred and fifty-two patients were eligible to be matched from the total of 237 male patients, who had been admitted to the BU; thus these patients (152 out of 237), addicted and non-addicted, were equally matched for age and other primary factors contributing to burn injury (Table 1 ).

All patients were male in the matched groups. Mean age was $33.27 \pm 13.88$ and mean LOS was $18.36 \pm 18.27$ days for the matched groups. Thirty-three out of 237 (13.9\%) patients of the unmatched group died at the BU including 23 (9.7\%) addicted patients and 10 (4.2\%) non-addicted patients (Table 1). Fourteen out of 152 (9.21\%) patients of the matched groups died at the BU including five (6.58\%) addicted patients and nine (11.84\%) non-addicted patients (Table 1).

In the matched groups, the McNemar test found statistically significant differences in mortality between the groups $(\mathrm{P}=0.000)$. The odds ratio for mortality was 0.524 (CI 95\%: 0.167-1.64) times greater for addicted patients compared with non-addicted patients. However Log Rank (Mantel-Cox) (Chi-Square $=1071 ; \mathrm{df}=1 ; \mathrm{P}=0.190$ ) didn't show significance differences between the two curves. Survival analysis using the Kaplan-Meier method revealed that the estimated mean of survival time was 59.02 (CI 95\%: 41.83 - 76.21) for non-addicted patients and 118.78 (CI 95\%: 106.52 - 131.04) for addicted patients. Given that, there was no overlap in the confidence intervals, it is likely that there was much difference in the "average" survival time. Morbidity-related factors didn't differ significantly between the two groups (Table 2).

In the unmatched group of the study population ( $\mathrm{n}$ = 237), thirty-three patients (14\%) died at the burn unit (BU), and 121 (51.0\%) were addicted patients. Two hundred and twenty-nine (97\%) patients had full-thickness burn wounds. Twenty-two (15\%) patients had total burn surface area (TBSA) of more than 50 percent. Gas explosion was the most common factor that caused burn in 78 (33\%) patients, 14 (6\%) of whom died. One hundred and seventeen (77\%) patients were less than 40 years old. The odds ratio was derived from the contingency table for variables, which were significantly associated with mortality (Table 3). Regarding mortality among addicted patients in the unmatched group, those who were separated, unemployed or had a psychoactive drug history, had significantly higher mortality rates than others $(\mathrm{P}<0.05)$ (Table 3$)$.

\section{Discussion}

Opium in burn patients could be identified as a factor against mortality, and it deserves to be studied further, despite the fact that addicted patients have not shown higher morbidity than non-addicted patients either.

A different result was obtained by Salehi et al., who compared mortality between two non-homogeneous nonequivalent drug-user and non-drug-user groups (13.23\% vs. $15.55 \%$ respectively), revealing that no significant differences existed between these groups (9). The odds ratio being equal to $0.524(\mathrm{OR}<1)$ indicated that drug dependency in burn patients could be identified as a preventive factor against mortality (15). In contrast, burn patients with positive blood alcohol levels on admission showed a significantly higher mortality rate $(31.5 \%)$ in comparison with the mortality rate $(18.1 \%)$ of patients with a negative blood alcohol level (7), thus opium-dependency might play a different role than alcohol dependency among burn patients. Further studies are needed to assess the impact of the effects of each substance on mortality.

Overall mortality rate (14.0\% of 237) was approximately equal to studies including (15\%) researches from Germany (16), (14.0\%) Pakistan (17), (18.8\%) Tabriz city of Iran and (14.77\%) Tehran city of Iran (9), which supports homogeneity regarding final outcome of survival and therapeutic measures among studies. It is also worth mentioning that mortality rate has been reported lower in developed countries as (7.0\%) in the US (6) and (1.4 - 18\%) in Europe (18).

Regarding morbidity, although complications in burn patients with opium addiction were not significantly higher (Table 2) yet the higher morbidity in addicted patients has been supported by several studies. One study indicated that substance-abusing patients show more complications (1.7 times) than others, as well as longer length of stay (1.8 times) for addicted patients (13). In Iran, one study reported higher length of stay for addicted patients (1.15 times) (9) and another study demonstrated length of saty among addicted patients was also higher than others (1.88 times) (8). In this way, Thombs et al. argued that length of stay for addicted patients was increased up to 1.2 times (6). 
Table 1. Characteristics of the Study Population and Matched Groups Based on Drug Dependency

\begin{tabular}{|c|c|c|c|c|c|c|c|}
\hline \multirow[t]{3}{*}{ Variables } & \multirow{2}{*}{\multicolumn{2}{|c|}{$\begin{array}{c}\text { Unmatched Group } \\
\operatorname{All}(\mathrm{N}=237)\end{array}$}} & \multicolumn{5}{|c|}{ Matched Groups } \\
\hline & & & \multicolumn{2}{|c|}{ Addicted $(\mathrm{N}=76)$} & \multicolumn{2}{|c|}{ Non-Addicted ( $N=76$ ) } & \multirow[t]{2}{*}{ P Value } \\
\hline & $\operatorname{mean} \pm \mathbf{S D}$ & $\%$ & $\operatorname{mean} \pm \mathbf{S D}$ & $\%$ & mean $\pm \mathbf{S D}$ & $\%$ & \\
\hline Age (yr) & $34.26 \pm 14.79$ & & $34.23 \pm 11.44$ & & $32.31 \pm 15.98$ & & 0.39 \\
\hline Marital status (separated) & & 8.0 & & 15.8 & & 17.1 & 0.86 \\
\hline Employment (unemployed) & & 27.0 & & 11.8 & & 15.1 & 0.23 \\
\hline TBSA burn & $34.38 \pm 24.90$ & & $32.26 \pm 21.83$ & & $31.52 \pm 21.65$ & & 0.83 \\
\hline Delay to admit (hour) & $12.08 \pm 2.25$ & & $11.27 \pm 41.46$ & & $14.25 \pm 29.17$ & & 0.61 \\
\hline Length of stay (days) & $16.14 \pm 17.16$ & & $20.18 \pm 21.07$ & & $16.55 \pm 14.88$ & & 0.22 \\
\hline Mortality & & 13.9 & & 3.3 & & 5.9 & 0.40 \\
\hline Crime history & & 8.6 & & 9.4 & & 0.0 & $0.00^{\mathrm{a}}$ \\
\hline Addiction history (year) & $5.13 \pm 8.49$ & & $8.60 \pm 8.42$ & & 0.0 & & $0.00^{\mathrm{a}}$ \\
\hline Psychiatric medications history & & 15.0 & & 9.9 & & 3.3 & $0.00^{\mathrm{a}}$ \\
\hline Suicide history & & 11.0 & & 11.0 & & 0.0 & $0.00^{\mathrm{a}}$ \\
\hline Full-thickness burn & & 98.0 & & 50.0 & & 48.7 & 0.13 \\
\hline Inhalation burn & & 10.0 & & 4.0 & & 5.3 & 0.38 \\
\hline Domestic gas & & 32.9 & & 22.4 & & 17.1 & 0.42 \\
\hline Flame & & 22.4 & & 10.5 & & 16.4 & 0.42 \\
\hline
\end{tabular}

Abbriviation: TBSA, total body surface area.

Abbriviation: IBSA, total body surface area.
${ }^{\mathrm{a}}$ Significant results were reported $(\mathrm{P}<0.05)$.

Table 2. Therapeutic Characteristics of the Study Population and Matched Groups Based on Drug Dependency

\begin{tabular}{|c|c|c|c|c|c|c|c|}
\hline \multirow[t]{2}{*}{ Variables } & \multicolumn{2}{|c|}{ Unmatched Group $(\mathrm{N}=237)$} & \multicolumn{2}{|c|}{ Matched Addicted $(\mathrm{N}=76)$} & \multicolumn{2}{|c|}{ Matched Non-Addicted $(\mathrm{N}=76)$} & \multirow[t]{2}{*}{ P Value } \\
\hline & mean $\pm S D$ & $\%$ & mean \pm SD & $\%$ & mean $\pm S D$ & $\%$ & \\
\hline Transfusion (units) & $2.64 \pm 4.76$ & & $2.64 \pm 4.45$ & & $3.29 \pm 5.61$ & & 0.432 \\
\hline CPR attempts & & 10.0 & & 3.4 & & 3.4 & 0.619 \\
\hline ICU stay (days) & $0.25 \pm 1.49$ & & $0.078 \pm 0.39$ & & $0.49 \pm 2.50$ & & 0.157 \\
\hline Surgery & & 40.0 & & 22.0 & & 18.0 & 0.619 \\
\hline Debridement & $0.89 \pm 1.43$ & & $0.88 \pm 1.42$ & & $0.98 \pm 1.41$ & & 0.650 \\
\hline Ventilator support (days) & $0.24 \pm 1.45$ & & $0.36 \pm 2.35$ & & $0.14 \pm 0.74$ & & 0.455 \\
\hline
\end{tabular}

Abbriviations: CPR, cardiopulmonary resuscitation; ICU, intensive care unit

These results support our findings, which showed that the length of stay, as a remarkable criterion of morbidity, for addicted patients was higher (1.4 times) than the other patients.

Age and TBSA were significant predictors for mortality, and this has been supported by several studies $(8,6$, 18). In addition, psychosocial factors could play an important role in mortality of burn patients (Table 3 ). Being separated, unemployed or having a psychoactive medication history indicated that these patients are psychologically severely unstable, resulting in poor commitment to recovery and worse outcomes. In fact, these kinds of factors could give clinicians a clue on how to identify psychologically complicated patients. Thus it is essential to recognize vulnerable patients and prepare extensive psycholog- ical support for them. A few studies have addressed that it is necessary to conduct early recognition and treatment of patients with in-hospital psychological distress (19, 20 , 21). As can be seen from Table 3 , an odds ratio greater than one indicates that exposure may increase the odds of death among burn patients who are psychologically complicated (22). It is recommended that psychological load of burn patients should be considered as a strong predictor of mortality.

Fifty-one percent of patients in the unmatched groups were addicted patients. This is higher than what has been reported by other studies (33\%) from the Tehran (9) (9.5\%) and Tabriz cities of Iran (11) and (3.3\%) the US (6), and signifies the extent to which drug dependency among burn patients is critical. Our sample didn't include female burn 
Table 3. Odds Ratios for Variables in Association with Mortality in the Unmatched Group of the Study Population $(\mathrm{N}=237)^{\mathrm{a}}$

\begin{tabular}{|c|c|c|}
\hline Variables & Odds Ratio & (95\% Confidence Interval) \\
\hline Marital status (separated) ${ }^{\mathbf{b}}$ & 3.24 & $(1.13-9.26)$ \\
\hline $\begin{array}{l}\text { Employment } \\
\text { (unemployed) }\end{array}$ & 2.77 & $(1.29-5.96)$ \\
\hline Substance dependency ${ }^{\mathbf{b}}$ & 2.46 & $(1.11-5.43)$ \\
\hline $\begin{array}{l}\text { Psychoactive medications } \\
\text { history }^{b}\end{array}$ & 4.93 & $(2.12-11.45)$ \\
\hline Suicide history ${ }^{b}$ & 3.62 & $(1.41-9.30)$ \\
\hline Marital status (separated) ${ }^{c}$ & 4.58 & $(1.37-15.34)$ \\
\hline $\begin{array}{l}\text { Employment } \\
{\text { (unemployed })^{\mathrm{c}}}^{\mathrm{c}}\end{array}$ & 3.66 & $(1.39-9.62)$ \\
\hline $\begin{array}{l}\text { Psychoactive medications } \\
\text { historyc }\end{array}$ & 4.36 & $(1.65-11.53)$ \\
\hline
\end{tabular}

${ }^{\mathrm{a}}$ Only significant results were reported $(\mathrm{P}<0.05)$.

${ }^{\mathrm{b}}$ All patients in the unmatched group were included.

' Only addicted patients in the unmatched group were included.

patients, who have lower rates of drug abuse. It is also important to mention that safety measures need excessive attention among addicted patients.

One limitation of this study was that burn patients were diagnosed only based on patients' self-disclosure and the researchers didn't verify claims with diagnostic tests for drug dependency to consider ethical and legal issues. Addiction in burn patients could be considered as a preventive factor against mortality that deserves further studies. Besides addicted patients didn't significantly show higher morbidity than non-addicted patients.

\section{Acknowledgments}

This study was supported by the vice chancellor for research of Mashhad University of Medical Sciences (Grant no. 920011). We gratefully acknowledge the staff and patients of the burn unit of Imam Reza hospital

\section{Footnotes}

Authors' Contributions: Mohammad Motamedolshariati: study concept and design analysis, study supervision, administrative, technical and material support; Amir Mirhaghi: drafting of the manuscript, interpretation of data and statistical analysis; Seyed Hassan Tavousi: acquisition of data and critical revision of the manuscript for important intellectual content.

Conflict of Interests: The authors declare that there was no conflict of interest regarding the publication of this paper.

\section{References}

1. Sadeghi-Bazargani H, Mohammadi R. Epidemiology of burns in Iran during the last decade (2000-2010): review of literature and methodological considerations. Burns. 2012;38(3):319-29. doi: 10.1016/j.burns.2011.09.025. [PubMed: 22119445].

2. Kooshyar H, Mirhaghi Saatchi A, Amoozegar M, Esmaeili H. Comparison between methods of assesment of burn wound infection. Evid Based Care J. 2008;19:7-16.

3. Motamed Shariati SM, Mirhaghi A. A comparison of burn size estimation methods' accuracy applied by medical students. Future of Med Edu J. 2014;4(1):36-40.

4. Hop MJ, Polinder S, van der Vlies CH, Middelkoop E, van Baar ME. Costs of burn care: a systematic review. Wound Repair Regen. 2014;22(4):43650. doi: 10.1111/wrr.12189. [PubMed: 25041616].

5. Zayakova Y, Vajarov I, Stanev A, Nenkova N, Hristov H. Epidemiological analysis of burn patients in east Bulgaria. Burns. 2014;40(4):683-8. doi: 10.1016/j.burns.2013.08.016. [PubMed: 24035580].

6. Thombs BD, Singh VA, Halonen J, Diallo A, Milner SM. The effects of preexisting medical comorbidities on mortality and length of hospital stay in acute burn injury: evidence from a national sample of 31,338 adult patients. Ann Surg. 2007;245(4):629-34. doi: 10.1097/01.sla.0000250422.36168.67. [PubMed: 17414613].

7. Haum A, Perbix W, Hack HJ, Stark GB, Spilker G, Doehn M. Alcohol and drug abuse in burn injuries. Burns. 1995;21(3):194-9. [PubMed: 7794500].

8. Maghsoudi H, Raghifar R. An aetiological survey of burns in abusers of various kinds of drugs admitted to the tabriz sina hospital burns ward in iran. Ann Burns Fire Disasters. 2010;23(4):186-93. [PubMed: 21991223].

9. Salehi SH, As'adi K, Musavi J, Ahrari F, Nemazi P, Kamranfar B, et al. Assessment of substances abuse in burn patients by using drug abuse screening test. Acta Med Iran. 2012;50(4):257-64. [PubMed: 22592576].

10. Moghanibashi-Mansourieh A, Deilamizade A. The state of data collection on addiction in Iran. Addiction. 2014;109(5):854. doi: 10.1111/add.12505. [PubMed: 24606041].

11. Hashmi M, Kamal R. Management of patients in a dedicated burns intensive care unit (BICU) in a developing country. Burns. 2013;39(3):493-500. doi: 10.1016/j.burns.2012.07.027. [PubMed: 22939740].

12. Goltsman D, Li Z, Bruce E, Maitz PK. Geospatial and epidemiological analysis of severe burns in New South Wales by residential postcodes. Burns. 2014;40(4):670-82. doi: 10.1016/j.burns.2013.09.005. [PubMed: 24290854].

13. Kelley D, Lynch JB. Burns in alcohol and drug users result in longer treatment times with more complications.J Burn Care Rehabil. 1992;13(2 Pt 1):218-20. [PubMed: 1587921].

14. Rich JT, Neely JG, Paniello RC, Voelker CC, Nussenbaum B, Wang EW. A practical guide to understanding Kaplan-Meier curves. Otolaryngol Head Neck Surg. 2010;143(3):331-6. doi: 10.1016/j.otohns.2010.05.007. [PubMed: 20723767].

15. Holcomb WJ, Chaiworapongsa T, Luke DA, Burgdorf KD. An odd measure of risk: use and misuse of the odds ratio. Obstet Gynecol. 2001;98(4):685-8. [PubMed: 11576589].

16. Theodorou P, Xu W, Weinand C, Perbix W, Maegele M, Lefering $\mathrm{R}$, et al. Incidence and treatment of burns: a twenty-year experience from a single center in Germany. Burns. 2013;39(1):49-54. doi: 10.1016/j.burns.2012.05.003. [PubMed: 22673118].

17. Iqbal T, Saaiq M, Ali Z. Epidemiology and outcome of burns: early experience at the country's first national burns centre. Burns. 2013;39(2):358-62. doi: 10.1016/j.burns.2012.07.011. [PubMed: 22867734 ]. 
18. Brusselaers N, Monstrey S, Vogelaers D, Hoste E, Blot S. Severe burn injury in Europe: a systematic review of the incidence, etiology, morbidity, and mortality. Crit Care. 2010;14(5):R188. doi: 10.1186/cc9300. [PubMed: 20958968].

19. Fauerbach JA, McKibben J, Bienvenu OJ, Magyar-Russell G, Smith MT, Holavanahalli R, et al. Psychological distress after major burn injury. Psychosom Med. 2007;69(5):473-82. doi: 10.1097/psy.0b013e31806bf393. [PubMed: 17585064].
20. De sousa A, Sonavane S, Kurvey A. Psychological issues in adult burn patients. Delhi Psy J. 2013;16(1):24-33.

21. Tedstone JE, Tarrier N, Faragher EB. An investigation of the factors associated with an increased risk of psychological morbidity in burn injured patients. Burns. 1998;24(5):407-15. [PubMed: 9725679].

22. Szumilas M. Explaining odds ratios. J Can Acad Child Adolesc Psychiatry. 2010;19(3):227-9. [PubMed: 20842279]. 